Original Research Paper

\title{
Quercetin Promotes the Expression of Genes Involved in Phagocytosis in Bovine Neutrophils
}

\author{
${ }^{1}$ Suphakit Srikok, ${ }^{1}$ Sukij Nambut, ${ }^{1}$ Kanruethai Wongsawan and ${ }^{1,2}$ Phongsakorn Chuammitri \\ ${ }^{I}$ Department of Veterinary Biosciences and Public Health, \\ Faculty of Veterinary Medicine, Chiang Mai University, Chiang Mai 50100, Thailand \\ ${ }^{2}$ Excellent Center in Veterinary Biosciences (ECVB), Chiang Mai University, Chiang Mai 50100, Thailand
}

Article history

Received: 09-05-2017

Revised: 27-06-2017

Accepted: 22-07-2017

Corresponding Author: Phongsakorn Chuammitri Department of Veterinary Biosciences and Public Health, Faculty of Veterinary Medicine, Chiang Mai University, Chiang Mai 50100, Thailand

E-mail:phongsakorn@gmail.com

\begin{abstract}
This study was conducted to explore the effect of Quercetin $(\mathrm{QH})$ on phagocytosis of bovine neutrophils. Neutrophils were isolated from fifteen multiparous Holstein cows. We in vitro treated neutrophils with PBS or $50 \mu \mathrm{M}$ of Quercetin (QH) or Cytochalasin B (CytB), as phagocytosis inhibitor, prior to monitoring phagocytosis of Escherichia coli by flow cytometry and microscopic examination. Additionally, the expressions of CORO1A, CYBA (gp91 ${ }^{\text {phox }}$ ), LAMP1, RAB7A, RAC1 and $P A K 1$ mRNA were analyzed by real-time PCR. In the time-course experiment, treated neutrophils were allowed to co-cultured with live bacteria for 30,60 and $90 \mathrm{~min}$ before measuring gene expressions. The expression levels of $I L-1 \beta$ and $T N F$ genes in order to demonstrate the antiinflammatory property of quercetin were assessed by conventional RTPCR. The results of flow cytometry and microscopic examination showed that the percentage of neutrophils performing phagocytosis were significantly higher in $\mathrm{QH}$ group in comparison with other groups. We reported here that mRNA expressions of COROIA, CYBA, LAMPI, RAB7A, RACl and $P A K 1$ genes involved in phagocytosis, were significantly up-regulated in $\mathrm{QH}$ group. As expected, CytB group had profound down-regulation of genes with one exception in PAK1. From our observations, the expression levels of phagocytic process genes in the $\mathrm{QH}$ group were optimum at $60 \mathrm{~min}$ and started to decline at $90 \mathrm{~min}$ of incubation. The data also indicated that quercetin inhibited inflammation by reducing the expressions of both $I L-1 \beta$ and $T N F$. In conclusion, the information in our experiments conclude that quercetin has the ability to boost the expression of genes involved in phagocytosis while reduces the expression of proinflammatory genes.
\end{abstract}

Keywords: Bovine, Neutrophil, Quercetin, Phagocytosis, Gene Expression

\section{Introduction}

The rapid and efficient innate defense mechanisms to resist microbial invasion may contribute to the overall health and well-being of human and animals. In innate immunity, neutrophils are actively phagocytic cells performing host defense through a variety of effector functions, i.e., Reactive Oxygen Species (ROS) generation, phagocytosis, degranulation and Neutrophil Extracellular Traps (NETs) release (Amulic et al., 2012; Brinkmann et al., 2004; Chuammitri et al., 2015). Neutrophil phagocytosis is a process, in which a large particle, such as bacteria, immune complex or apoptotic cell is being intake into this cell (Esmann et al., 2010; Grinberg et al., 2008). Innate immune cells; macrophages, neutrophils and dendritic cells (DCs) possess this effector function. Phagocytosis of human Polymorphonuclear leukocytes (PMNs) was assisted by the Fc receptor and Complement Receptors (CRs) led to the expression of many involving genes in cytokine production and signal transduction (Kobayashi et al., 2002). The gene expression patterns differ when phagocytosis is examined during the 90 min-interval as the genes were either induced or repressed (Kobayashi et al., 2002). The available information stated that gene expression and cell signaling pathways involved in phagocytosis, AMP-activated Protein Kinase (AMPK), may result in anti-inflammatory in phagocytosis of $E$. coli, as well as, the changes in cytoskeletal reorganization (Bae et al., 2011). The AMPK signaling also stimulated $P A K 1 / 2$ genes and moreover the $R A C 1$ gene that helped reduce the expression of other genes 
involved in the inflammatory process, such as $T N F-\alpha$ in neutrophils via the induction of Toll Like Receptor 4 (TLR4) (Bae et al., 2011). The microarray study of human PMN gene expression during bacterial Phagocytosis revealed the global changes of many key gene transcripts (Kobayashi et al., 2003). The genes in associated with the inflammatory response and apoptosis regulators i.e., $I L-1 \alpha$, $I L-1 \beta, I L-1 \varepsilon, I L-1 R N, I L-8, I L-10, I L-12 \beta, I L-15, T N F \alpha$, Vascular Endothelial Growth Factor (VEGF), OncostatinM $(O S M), I L-6, G R O \beta$ and $G R O \gamma$ were up-regulation as shown and reviewed by (Kennedy and DeLeo, 2009; Kobayashi et al., 2003; 2005).

At present, the role of herbs and natural substances classified as one option to help strengthen the immune system of cattle. One of spotlight herbal phenolic substances is quercetin, which is found in many edible plants, fruits and vegetables (Erlund, 2004; Formica et al., 1995). The bioavailability studies of quercetin and its metabolites; aglycone and rutin, in cows demonstrated that quercetin can be absorbed via digestive tracts and eventually entered the plasma (Berger et al., 2012; Gohlke et al., 2013; Maciej et al., 2015). Several in vivo studies of quercetin in neonatal calves, periparturient and lactating cows, in the aspects of bovine health and production, have been recently reported (Gohlke et al., 2013; Maciej et al., 2015; Stoldt et al., 2015); however, another point of view remains scarce in bovine innate immunity.

Many published works have emphasized that quercetin also exerts its effects by promoting and acting as an antioxidant and anti-inflammation (Boots et al., 2008; Cho et al., 2003; Chuammitri et al., 2017; De Marchi et al., 2009; Nair et al., 2006). In fact, quercetin affects the mRNA gene expressions and associated proteins in the innate immune cells, such as the reduction of the IL-1 $\beta$ mRNA level during inflammation was documented (Valerio et al., 2009). We have previously demonstrated the in vitro effects of quercetin on bovine neutrophil phagocytosis of Escherichia coli (Chuammitri et al., 2015), but in great detail study of how gene cascades in bovine neutrophil phagocytic processes were regulated due to proposed quercetin effects is incompletely elucidated at molecular levels. To our knowledge, little is known about the action of quercetin on the dynamic of gene expressions during bacterial phagocytosis of bovine neutrophils. To continue examining the immunomodulatory action of anticipating quercetin effects, this study aims to investigate how quercetin alters the expressions of key phagocytosis genes; CORO1A, CYBA (gp91 ${ }^{\text {phox }}$ ), LAMP1, RAB7A, RAC1 and $P A K 1$ during bacterial phagocytosis.

\section{Materials and Methods}

\section{Reagent}

Quercetin Hydrate (QH) cat. No 337951, Cytochalasin B (CytB), Propidium Iodide (PI), Hanks'
Balanced Salt Solution without calcium and magnesium (HBSS), Citrate-Dextrose solution (ACD) and Trypan blue were purchased from Sigma-Aldrich, St. Louis, MO, USA. Fetal Bovine Serum (FBS), Gibco RPMI 1640 medium with phenol red and RNAlater were obtained from Life Technologies, Carlsbad, CA, USA.

\section{Ethical Approval}

The animal experimental procedure was approved to be conducted under the Animal Care and Use Ethics Committee (FVM-ACUC Ref. No: S5/2557).

\section{Animals, Blood Collection and Bovine Neutrophil Isolation}

Healthy, multiparous Holstein cows during nonlactating period were used as blood donors (Jinghui et al., 2014; Martinez et al., 2014). A total number of 15 cows in 3 independent experiments, 5 cows each, were recruited in the study. All dairy cows were housed at local dairy farms of Mae On district and Mae Hia Agricultural Research, Demonstration and Training Center. Whole blood was collected by jugular venipuncture into a sterile syringe containing $1 \times$ ACD solution. Neutrophil isolation protocol was previously described (Chuammitri et al., 2015). Finally, cell density was adjusted to approximately $2 \times 10^{6}$ cells per $\mathrm{ml}$ in RPMI 1640 medium supplemented with $1 \%$ FBS.

\section{Quercetin}

Stock Quercetin solution at $5 \mathrm{mM}$ was prepared by dissolving dry quercetin powder in $95 \%$ ethanol and filter sterile. Working solution at $50 \mu \mathrm{M}$ concentrations was freshly prepared on the day of the experiment, protected from light and stored at room temperature until use. The $50 \mu \mathrm{M}$ of quercetin showed no in vitro cytotoxicity to bovine neutrophils as previous report (Chuammitri et al., 2015).

\section{Escherichia coli Propagation and Opsonization}

Escherichia coli (E. coli) was isolated from clinical mastitis cow. The bacteria were freshly prepared by colony picking method and propagation in Luria-Bertani Broth (LB broth, Caisson Laboratories, North Logan, UT, USA). The bacterial number was adjusted to approximately $10^{8} \mathrm{CFU} / \mathrm{ml}$. Live E. coli was opsonized with $10 \%$ heat-inactivated normal bovine serum for 20 $\min$ at $37^{\circ} \mathrm{C}$ prior to use in the experiment.

\section{Fluorescent Staining of Bacteria}

Fluorescent $E$. coli was prepared by growing bacteria to $\log$ phase, heat killed $\left(70^{\circ} \mathrm{C}, 60 \mathrm{~min}\right)$, washed with PBS and centrifuged $(12,000 \mathrm{rpm}, 5 \mathrm{~min})$. Bacterial DNA pellet $\left(10^{8} \mathrm{CFU} / \mathrm{ml}\right)$ was stained with $5 \%$ Propidium Iodide (PI) in PBS and incubated at room temperature in the dark for $60 \mathrm{~min}$ (Keogh et al., 2011). 
PI labeled E. coli was adjusted to $10^{6} \mathrm{CFU} / \mathrm{ml}$ with PBS and stored at $4^{\circ} \mathrm{C}$ until use. Fluorescent E. coli was opsonized with $10 \%$ heat-inactivated normal bovine serum for $20 \mathrm{~min}$ at $37^{\circ} \mathrm{C}$ before using in phagocytosis assay.

\section{Phagocytosis}

The phagocytosis of PI labeled E. coli was assessed via flow cytometry (Della Libera et al., 2015). In brief, neutrophils $\left(2 \times 10^{5}\right.$ neutrophils $)$ were seeded into duplicate wells of a 96-well, flat bottom cell culture plate. Cells were treated with $50 \mu \mathrm{M}$ quercetin, or 10 $\mu \mathrm{g} / \mathrm{ml}$ cytochalasin B (CytB), in order to inhibit phagocytosis (Khatua et al., 2012), or PBS for $30 \mathrm{~min}$ at $37^{\circ} \mathrm{C}, 5 \% \mathrm{CO}_{2}$. Subsequently, opsonized fluorescent $E$. coli at MOI of 10 were then added to the cells, centrifuged and incubated $\left(37^{\circ} \mathrm{C}, 5 \% \mathrm{CO}_{2}\right)$ for $45 \mathrm{~min}$. Sample acquisitions (50,000 events) were performed by CyAn ADP High-Performance Flow Cytometer (Beckman Coulter), then analyzed by using the FlowJo 10 (Treestar, Ashland, OR, USA).

Enumeration of Phagocytosis of E. coli by Bovine Neutrophils under Light Microscope

Neutrophils $\left(2.5 \times 10^{4}\right.$ neutrophils $)$ were seeded into duplicate wells of a 96-well, flat cell culture plate. Cells were treated as previously described in phagocytosis assay. Next, live, opsonized fluorescent $E$. coli at MOI of 10 were then added to the cells, centrifuged and incubated for $45 \mathrm{~min}$. Cytospin slides were prepared from an aliquot of neutrophils after phagocytosis and seeded onto circular coverlips (15 $\mathrm{mm}$ diameter) that were placed into a 24 -well plate. Slides were fixed with absolute ethanol, stained with Dip Quick and examined with a Zeiss Axio Scope A1 (Carl Zeiss, Thornwood, NY, USA). Percentage of phagocytosis with different treatments were manually counted from 5 random fields (100 cells per field) at $10 \times$ objective by three well-trained observers in a blinded technique (Yan et al., 2012).

Gene Expression Studies by Quantitative RealTime Reverse Transcription (qRT-PCR) and Semi-Quantitative RT-PCR

Neutrophils $\left(1 \times 10^{6}\right.$ cells $)$ were seeded into duplicate wells of a 24-well cell culture plate. Then, cells were treated with PBS, quercetin, or CytB for 30 min before co-cultured with $10 \mathrm{MOI}$ of opsonized $E$. coli in serum-free RPMI-1640 medium for another 45 min. After incubation, cells from two wells were combined, washed and spun. Neutrophil pellet was resuspended in RNAlater. In the time-course experiment, the assay setting was nearly identical to the above stated procedures, but with some modifications. In particular, neutrophils were allowed to co-cultured with opsonized $E$. coli for 30,60 and $90 \mathrm{~min}$ at $37^{\circ} \mathrm{C}, 5 \% \mathrm{CO}_{2}$, respectively.

\section{RNA Extraction and Complementary DNA (cDNA) Synthesis}

Total RNA was extracted and purified by NucleoSpin RNA (Macherey-Nagel, Bethlehem, PA, USA) according to the provided protocols. Complementary DNA (cDNA) was reverse-transcribed from a starting amount of $2 \mu \mathrm{g}$ of total RNA by Tetro cDNA Synthesis Kit (Bioline, Taunton, MA, USA) and stored at $-20^{\circ} \mathrm{C}$ until further use.

\section{Primers for qRT-PCR and Semi-Quantitative RT- $P C R$}

Primers for real-time PCR; CORO1A, CYBA, $L A M P 1, R A B 7 A, R A C 1, P A K 1$ and $A C T B$ as endogenous control (Shirasuna et al., 2015) were designed using Primer3 Plus. Primers for $I L 1 B, T N F$ and GAPDH were previously reported (Chuammitri et al., 2015). The PCR primers were synthesized by Macrogen, Seoul, Korea. The sequence information of oligonucleotide primers used in this study is indicated in Table 1.

\section{Quantitative Real-Time Reverse Transcription PCR (qRT-PCR)}

The qRT-PCR was performed to investigate the effect of quercetin on phagocytosis related gene expressions in bovine neutrophils. The quantification of mRNAs was performed in triplicate on ABI 7300 RealTime PCR System equipped with SDS Software v1.4 (Life Technologies). The qRT-PCR was performed on 100 ng cDNA using SensiFAST SYBR Hi-ROX Kit (Bioline) as per manufacture's instruction. Subsequently, specificity was confirmed by dissociation curve analysis $\left(T_{m}\right)$. Correct product sizes were also determined by $2 \%$ agarose gel (Chuammitri et al., 2017). Analysis of relative gene expression was calculated from the $C_{t}$ of the gene of interest (target) and $A C T B$. The expression levels (fold difference) were reported using $2^{-\Delta \Delta C t}$ method (Livak and Schmittgen, 2001).

\section{Semi-Quantitative Reverse Transcription PCR (RT-PCR)}

Semi-quantitative RT-PCR was performed to investigate the effect of quercetin on $I L I B$ and $T N F$, as pro-inflammatory cytokine gene expressions in bovine neutrophils after phagocytosis and time-course induction of phagocytosis. PCR reactions were performed using MyTaq HS Red Mix (Bioline). The annealing temperature at $59^{\circ} \mathrm{C}(I L 1 B$ and $G A P D H)$ or $57^{\circ} \mathrm{C}(T N F)$ was set. The amplified PCR products were electrophoresed in $1.5 \%$ agarose gel. Gel band intensities were quantified by Gel Analyzer Options in ImageJ version 1.46r (Chuammitri et al., 2015). Expression levels are represented relative to GAPDH expression. 
Table 1. Details of PCR primer sequences

\begin{tabular}{|c|c|c|c|c|c|}
\hline Gene & Accession no. & Primer & Sequence & $\begin{array}{l}\text { Annealing } \\
\text { temp }\left({ }^{\circ} \mathrm{C}\right)\end{array}$ & $\begin{array}{l}\text { Product } \\
\text { size (bp) }\end{array}$ \\
\hline \multirow[t]{2}{*}{$\overline{C O R O I A}$} & NM_174521 & Forward & TGCCGTGTTTGTATCCGATG & 59 & 177 \\
\hline & & Reverse & AGGTAGACGATGTTGGTGTCAG & & \\
\hline \multirow[t]{2}{*}{$C Y B A$} & NM_174034 & Forward & TCAGTTCACCCAGTGGTACC & 59 & 135 \\
\hline & & Reverse & ACTCTGGTCAGGTACTTCTGTC & & \\
\hline \multirow[t]{2}{*}{$L A M P 1$} & NM_001075124 & Forward & ACAACGTTTCTGGCAGCAAC & 59 & 125 \\
\hline & & Reverse & GGTCTTGTTGGGGTTGACATTG & & \\
\hline \multirow[t]{2}{*}{$R A B 7 A$} & NM_001035081 & Forward & ACCATGCAGATTTGGGACAC & 59 & 126 \\
\hline & & Reverse & AAGGGTCTTGAACGTGTTGG & & \\
\hline \multirow[t]{2}{*}{$R A C l$} & NM_174163 & Forward & TGCCAATGTCATGGTGGATG & 59 & 193 \\
\hline & & Reverse & ACAATGGTGTCGCACTTCAG & & \\
\hline \multirow[t]{2}{*}{$P A K l$} & NM_001076898 & Forward & AAGGAACGGCCAGAGATTTCTC & 59 & 158 \\
\hline & & Reverse & TGCGGGTTTTTCTTCTGCTC & & \\
\hline \multirow[t]{2}{*}{$A C T B$} & NM_173979 & Forward & TGCGGCATTCACGAAACTAC & 59 & 146 \\
\hline & & Reverse & AGGGCAGTGATCTCTTTCTGC & & \\
\hline \multirow[t]{2}{*}{$I L 1 B$} & M35589 & Forward & CGTACCTGAACCCATCAACGAAAT & 59 & 564 \\
\hline & & Reverse & GGCGTATCACCTTTTTTCACACAA & & \\
\hline \multirow[t]{2}{*}{$T N F$} & Z14137 & Forward & TCTCAAGCCTCAAGTAACAAGCCG & 57 & 414 \\
\hline & & Reverse & CAGGTAGTCCGGCAGGTTGATCTC & & \\
\hline \multirow[t]{2}{*}{ GAPDH } & NM_001034034 & Forward & AGTTCAACGGCACAGTCAAG & 59 & 243 \\
\hline & & Reverse & TCACGCCCATCACAAACATG & & \\
\hline
\end{tabular}

\section{Heat Map of Gene Expressions}

Gene expression patterns from the average expression level of each gene presented in the form of a heat map of an average linkage method with Euclidean distance. Heat map was generated by Genesis. 1.7.6 (Sturn et al., 2002).

\section{Data Analysis}

The data were first screened for potential outlier by robust Regression and Outlier Removal (ROUT) methods. The normality test was done by the D'AgostinoPearson omnibus test prior to performing the unpaired twotailed Student $t$ test, one-way ANOVA or Kruskal-Wallis Test and two-way ANOVA. The statistical analysis was considered significant when $\mathrm{p}<0.05$. The results are reported as mean with standard error (mean $\pm \mathrm{SE}$ ). Information obtained from the statistical analyses is represented as graphs generated by GraphPad Prism (GraphPad software, San Diego, CA, USA).

\section{Results}

\section{Neutrophil Phagocytosis of E. coli by Flow Cytometry}

The ability of neutrophil phagocytosis under the supplementation of quercetin and phagocytosis inhibitor can be reflected by the amounts of fluorescently labelled bacteria being ingested or Median Fluorescence Intensity (MFI) as determined by flow cytometry. Neutrophils treated with quercetin was found to have the highest MFI when compared with PBS and CytB group (Fig. 1B and $1 \mathrm{C}, \mathrm{p}=0.018)$, with an average MFI in PBS $(20.77 \pm 1.82), \quad$ quercetin $(28.54 \pm 2.65)$ and CytB $(21.35 \pm 1.44)$, respectively.

\section{Phagocytosis under Light Microscope}

The mean percentages of phagocytosis as depicted in Fig. 2A-2B by neutrophils received PBS, quercetin and CytB revealed under light microscope that the ability of phagocytosis can be enhanced when supplementation of quercetin in the condition other than with PBS or an inhibitor (CytB). The average percentages of phagocytosis in PBS, quercetin and CytB experiment group was equivalent to $42.03 \pm 4.11,58.43 \pm 4.17$ and $39.94 \pm 3.13$, respectively Fig. $2 \mathrm{~B},(\mathrm{p}<0.005)$.

\section{Gene Expressions by Real-Time PCR}

We have previously reported that bovine neutrophils responded well for phagocytosis when supplemented with $50 \mu \mathrm{M}$ quercetin (Chuammitri et al., 2015). Here we further explored the effects of quercetin on the levels of gene expressions during and at the end of phagocytosis of E. coli. The results suggested that the expression of genes involved in that process after neutrophils has been supplemented with PBS, quercetin and CytB for $30 \mathrm{~min}$ and cells were allowed to intake opsonized bacteria for another $45 \mathrm{~min}$. The measurable gene expression after 45 min-interval on real-time PCR showed the expression of all tested genes; CORO1A $(\mathrm{p}<0.0001), C Y B A(\mathrm{p}<0.0001)$, LAMP1 ( $\mathrm{p}=0.0002), R A B 7 A(\mathrm{p}=0.0001), R A C 1$ $(\mathrm{p}<0.0001)$, PAK1 $(\mathrm{p}=0.4464)$, in the quercetin group had higher levels than that of either control (PBS) or CytB supplemented groups. Although the expression level of $P A K 1$ in the quercetin group was higher when compared to the control group, but the expression of this gene in CytB has increased as well, Fig. 3A. Unsupervised hierarchical clustering confirmed that the gene expression profile of quercetin-treated neutrophils helps increase the phagocytic activity Fig. 3B. 


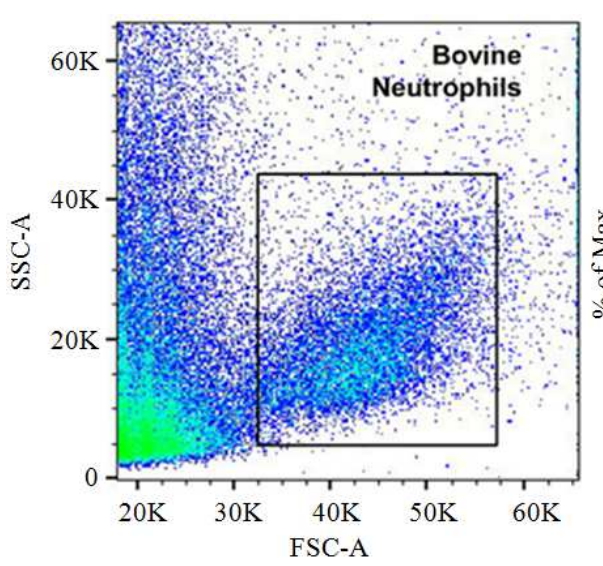

(A)

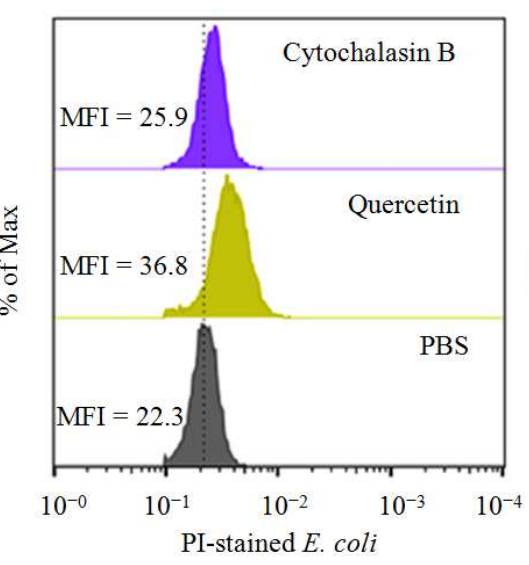

(B)

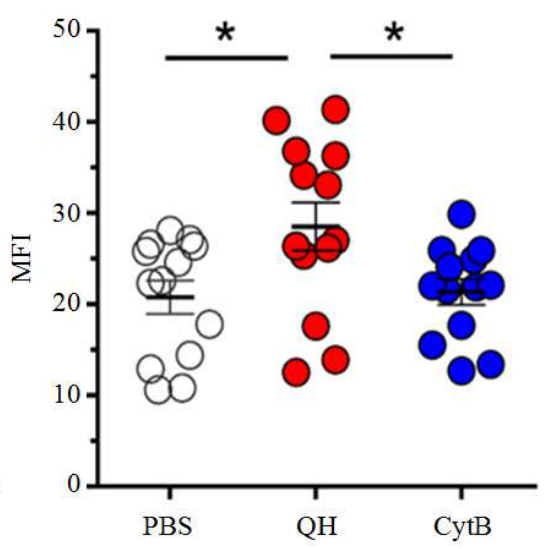

(C)

Fig. 1. Representative flow cytometry results of phagocytosis of PI-stained E. coli in bovine neutrophils. (A) Gating strategy of bovine neutrophils (B) Histograms shows Median Fluorescence Intensity (MFI) of each treatment (PBS, Quercetin (QH) and cytochalasin $\mathrm{B}(\mathrm{CytB})$ as inhibitor). (C) Each data point in scatter plots correspond to an individual cow ( $n=13$ each group). Data are mean $\pm \mathrm{SE}$, one-way ANOVA, * $\mathrm{p}<0.05$

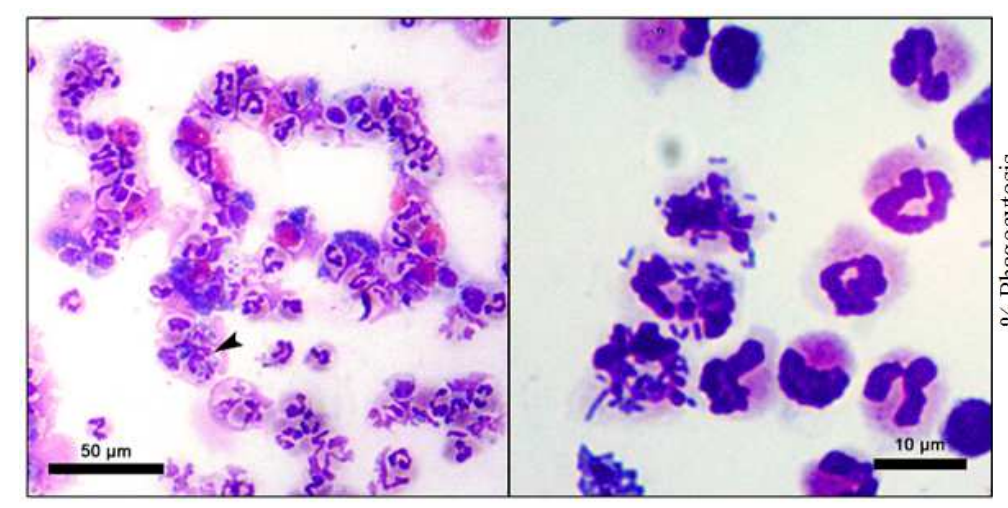

(A)

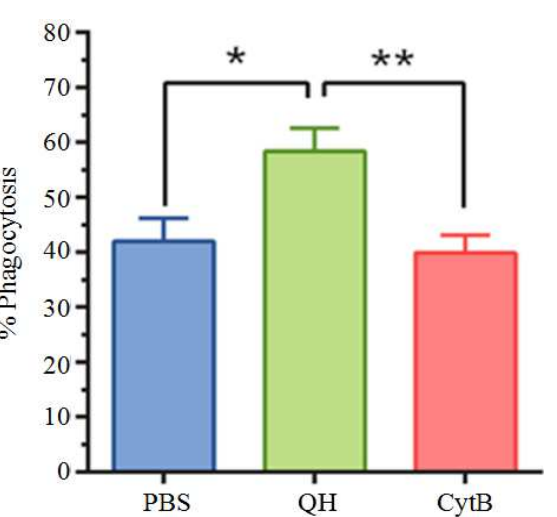

(B)

Fig. 2. Representative microscopic images of bovine phagocytosis of live E. coli under supplementation of quercetin. (A) Arrowhead

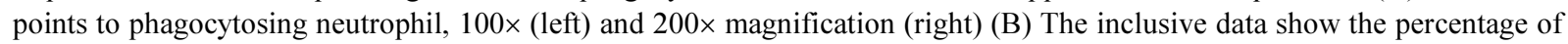
phagocytosis in PBS, Quercetin $(\mathrm{QH})$ and cytochalasin B (CytB) groups. Data are mean $\pm \mathrm{SE}$ of three independent experiments ( $n=11-13$ each group), one-way ANOVA, $* \mathrm{p}<0.05, * * \mathrm{p}<0.01$

\section{Time-Course of Gene Expressions}

As reported earlier in this section that quercetin enhanced genes the expression of the phagocytic process at the determining time (45 min), we asked that the dynamic of genes involved in that process may depend on contact time with quercetin treatment. Neutrophils were fortified with PBS or quercetin for 30, 60 and 90 min of allotted time. The expression of all studied genes after receiving quercetin have changed dramatically in quercetin as measured by real-time PCR Fig. 4. The data show that cells in the quercetin group had higher expression levels of CORO1A, LAMP1, RAB7A and RAC1 compared to the control group at 30 and $60 \mathrm{~min}$ of phagocytosis with the highest levels at 60-min Fig. 4A. The expression levels of aforementioned genes in the quercetin group were declined significantly in 90-min time. The gene expression data of $C Y B A$ and $P A K 1$ from cell treated with quercetin demonstrated the different patterns. The maximum expression of these two genes was found to be significantly increased at $30 \mathrm{~min}$ of incubation, but the levels were gradually decreased at 60 and 90 min-time, respectively Fig. 4A. A heat map of gene expression in quercetin groups at 30 to 60 min-time also depicted the increased in phagocytic activity, but later declined at 90 min-time Fig. 4B.

\section{Pro-Inflammatory Gene Expressions}

Effect of quercetin to reduce pro-inflammatory gene expressions in neutrophils being phagocytose $E$. coli was measured by reverse-transcription PCR. The expression of $I L I B$ and $T N F$ revealed a significant reduction of both $I L 1 B(\mathrm{p}=0.0004)$ and $T N F(\mathrm{p}=0.026)$ by quercetin compared with control Fig. 5. 

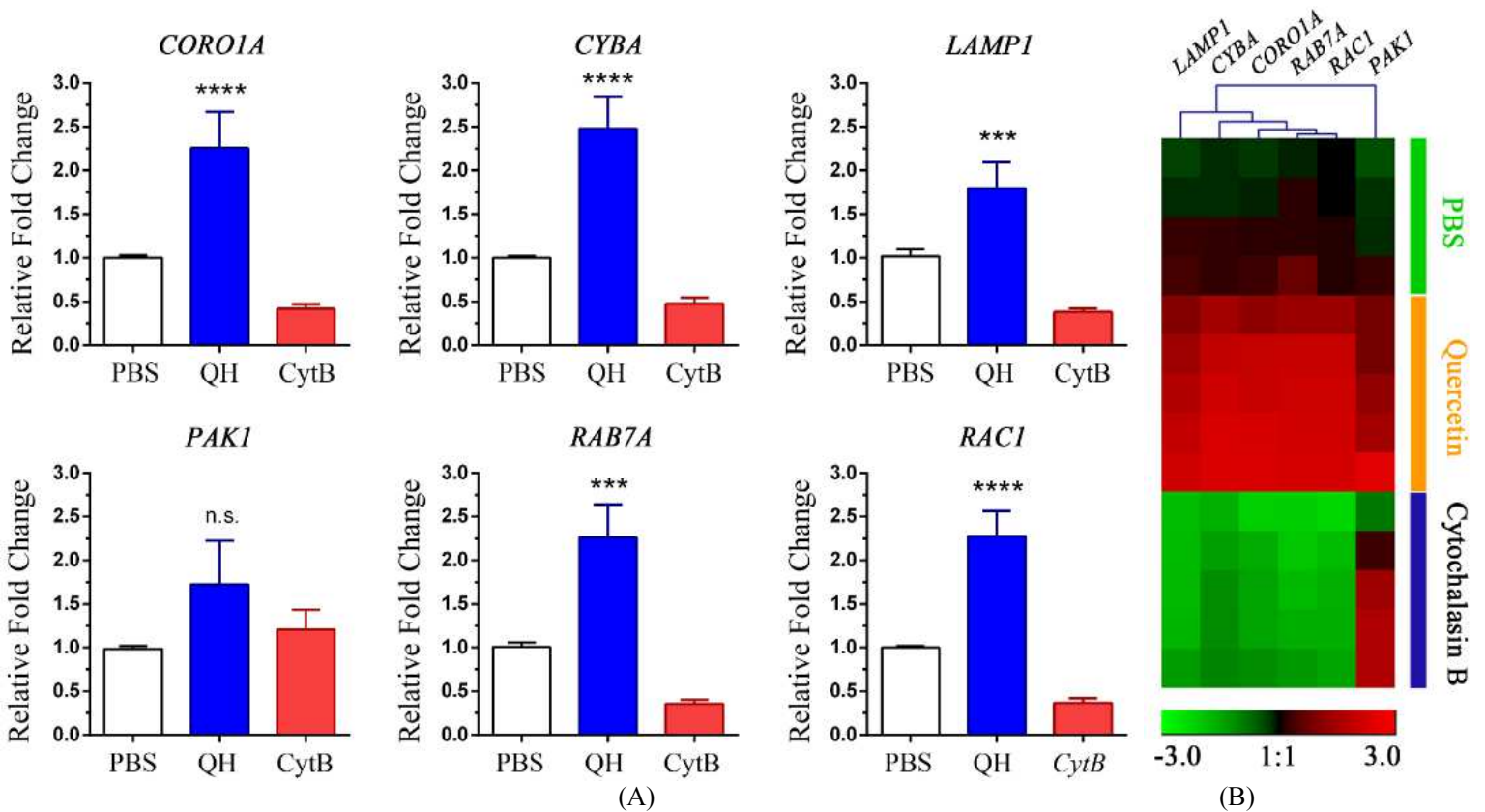

Fig. 3. Real-time RT-PCR analyses of bovine neutrophil genes involved in phagocytosis of E. coli. Top panel shows relative COROIA, CYBA, LAMP1 expression levels after normalization to $\beta$-actin expression in PBS, Quercetin $(\mathrm{QH})$ and cytochalasin $\mathrm{B}(\mathrm{CytB})$ groups after incubation with live E. coli for $45 \mathrm{~min}$. Bottom panel shows relative gene expressions of $P A K 1, R A B 7 A$ and $R A C 1$. The results are inclusive of three independent experiments represented as mean \pm SE $(n=7-8$ each group), one-way ANOVA, ${ }^{* * *} \mathrm{p}<0.001, * * * * \mathrm{p}<0.0001, \mathrm{n} . \mathrm{s}=$ no significance
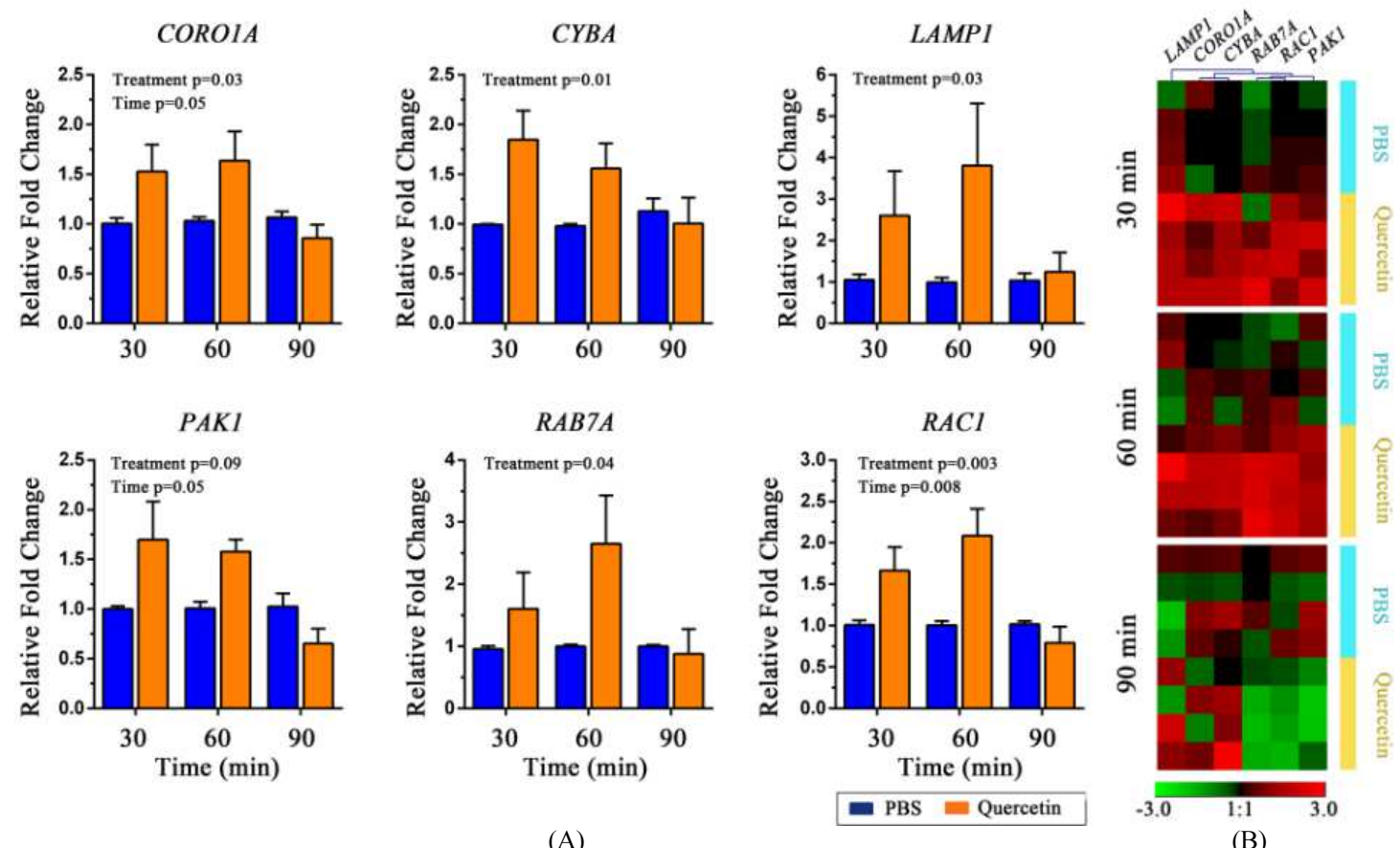

(A)

(B)

Fig. 4. Time-course differential gene expression levels in bovine phagocytosis by qPCR analyses. Top panel shows relative COROIA, CYBA, LAMPI expression levels after normalization to $\beta$-actin expression in PBS and quercetin groups after incubation with live $E$. coli for 30,60 and $90 \mathrm{~min}$. Bottom panel shows relative gene expressions of $P A K 1, R A B 7 A$ and $R A C l$. The results are inclusive of three independent experiments represented as mean $\pm \mathrm{SE}$ ( $n=9-12$ each group), Two-way ANOVA (treatment and time effect) 


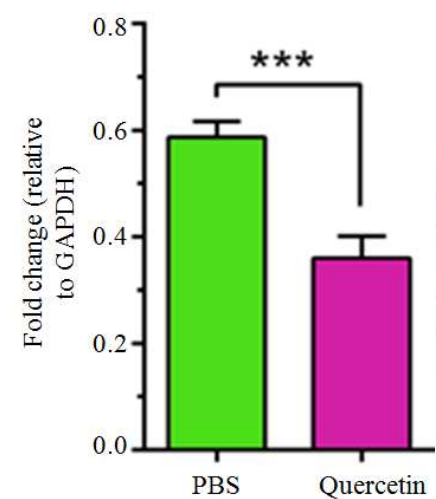

(A)

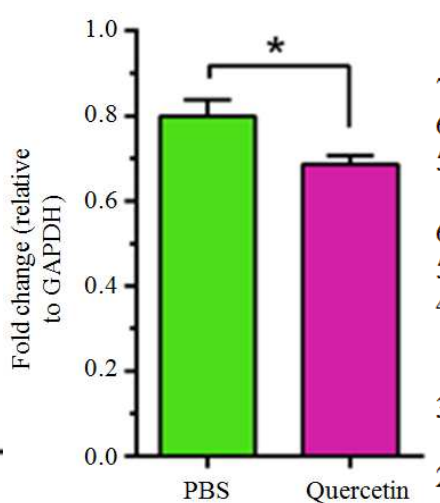

(B)

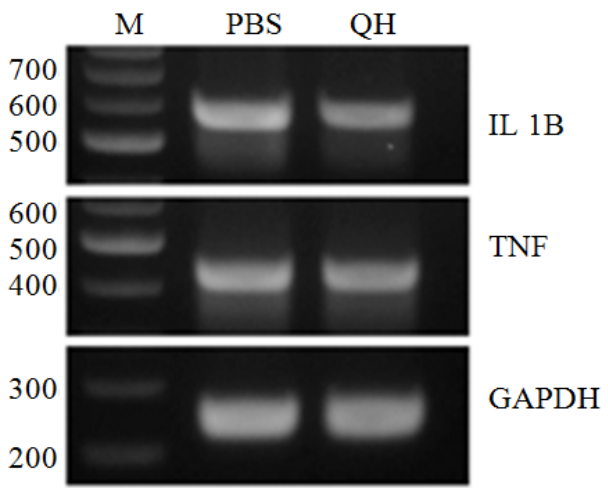

(C)

Fig. 5. Quercetin down-regulated the expression of $I L-1 \beta$ and $T N F$ genes in phagocytosis. (A-B) Relative densitometric analysis of $I L-1 \beta$ (A) and TNF (B) mRNA expression level compared to GAPDH. (C) Representative RT-PCR bands from PBS or quercetin treated neutrophils. Quantification of band intensities was performed using ImageJ. Data are expressed as mean \pm SE of two independent experiments ( $n=6-9$ per group). ${ }^{*} \mathrm{p}<0.05,{ }^{* * *} \mathrm{p}<0.001$ versus PBS by unpaired $t$-test

\section{Discussion}

Bovine neutrophils ingest bacterial pathogens by a process known as phagocytosis and the ingested microbes are destroyed by the combination of armors equipped within these cells. The present work has emphasized bovine neutrophil phagocytosis of E. coli under quercetin supplementation. Our results demonstrate that quercetin mitigates the expression levels of genes associated with inflammation, including $I L-1 \beta$ and $T N F$. Those results are consistent with the report that quercetin lowers the level of proinflammatory gene expression as a consequence of oxidative stress and different stimuli (Chuammitri et al., 2015; Valerio et al., 2009). Accordingly, the antiinflammatory action of quercetin on TNF- $\alpha$ is dosedependent manner (Nair et al., 2006).

Based on the results of recent publication by Chuammitri et al. (2017), it could be considered that quercetin might exert its anti-inflammatory effects of mitigating of either proinflammatory genes $(I L 1 B, I L 6$, $T N F)$ or microRNAs (MIR24-2, MIRI46A and MIR181C). Our results in a reduction of IL1 $\beta$ and TNF expressions by action of quercetin agreed with aforementioned study. Even though the two experiment settings used quercetin at $50 \mu \mathrm{M}$ final concentration, the course of inflammatory processes might originate from diverse pathways. In fact, both bacterial cells (in the current study) and cell wall component (LPS) have one thing in common to initiate inflammatory signals, nevertheless, quercetin could possibly lessen proinflammatory gene expressions via inhibition of key transcription factors (Boesch-Saadatmandi et al., 2011). These phenomena could emphasize the important property of quercetin in specifically targeted of transcription factors.

Fundamentally, the transcription factor, NF- $\kappa \mathrm{B}$ has played a pivotal role in mediation of genes related to the inflammatory process, such as $I L-1 \beta, I L-6$ or $i N O S$
(Bliss et al., 2005; Kobayashi et al., 2002). Furthermore, the increased expressions of many genes involved in phagocytosis are observed during the first few hours after induction (Kobayashi et al., 2002). This phenomenon may be controlled by the transcription factor NF-kB. Many reports have mentioned that quercetin exerts its modulatory effects by reduction of pro-inflammatory genes through NF- $\mathrm{BB}$ inactivation (Boesch-Saadatmandi et al., 2011; Cho et al., 2003; Nair et al., 2006). From previous studies, quercetin inhibits the production of many proinflammatory cytokines, including IL-1 $\beta$ and TNF, the findings from the present work suggest that quercetin may be able to modulate the immune response via the NF-kB pathway.

In the experimental mouse model, supplementation of quercetin and exploratory studies on mRNA expressions of $I L-6$, C-Reactive Protein (CRP), Monocyte Chemoattractant Protein 1 (MCP-1) and Acyloxyacyl Hydrolase $(A O A H)$ were irrelevant between the amount of quercetin intake and the expression levels of genes (Boesch-Saadatmandi et al., 2012). This study intends to demonstrate the effects of quercetin on both the influence of phagocytosis and phagosome formation. In order to select the representative genes involved in the process of phagocytosis to be monitored, we have used the KEGG pathway analysis as a searching tool. We have focused on CORO1A (coronin), CYBA (gp91 ${ }^{\text {phox }}$ ), $L A M P 1, P A K 1, R A B 7 A$ and $R A C 1$, which are illustrated as our candidate genes. The effects of quercetin have securely confirmed our prediction that quercetin is truly induced those genes during the phagocytosis in bovine neutrophils of live $E$. coli bacteria.

Many published works indicate the actual quercetin effects on gene expression are linked to the exposure time (Cho et al., 2003; Liu et al., 2005). Our results also show that most gene expression levels increase from 30 to $60 \mathrm{~min}$ of the phagocytic period by quercetin actions. 
Thus, our results are consistent with a report in the macrophage cells (J774.A1) in which the changes in expression levels of gene transcription and related proteins (Hoffmann et al., 2010). In that particular study, the increase production of gene transcriptions and protein productions i.e., Lamp1/2, Rab7 were intensified during 30 to $60 \mathrm{~min}$ of incubation (Hoffmann et al., 2010; Zhang et al., 2005). In this study, the data provide the information that the expression of CORO1A, LAMP, $R A B 7 A$ and $R A C 1$ are all obviously increased during phagocytosis. Therefore, it may be concluded that quercetin might affect both the initial and terminal stage of phagocytosis of bovine neutrophils. In addition to the above-mentioned genes, there were some known correlations of activated genes in phagocytosis; $\mathrm{Cdc} 42$, Racl and Rho, that potentially triggering and may have been associated with exposure time (Zhang et al., 2005). The F-actin polymerization, as part of internalization and the formation of the phagosome, is controlled by $P A K$ gene. This structure could be disrupted by cytochalasin $\mathrm{B}$ treatment in which actin polymerization are seized (Bao et al., 2012; Berends et al., 2010; Zhang et al., 2005). In this study, when bovine neutrophils were applied with cytochalasin B, the phagocytic activities of this cell were retarded as measurement by flow cytometry, microscopy or even with the gene expression profiling. The observed data are in accordance with earlier studies (Bao et al., 2012; Berends et al., 2010).

The engaging genes of phagocytosis in quercetin treated neutrophils tend to maximize most of its expression levels at 60 -min interval. After reaching the maximum, it begins to decline after $90 \mathrm{~min}$. This observation is inconsistent with previous study (Kobayashi et al., 2002). In that report, the expression studies on receptor-mediated phagocytosis of human PMNs were reached the highest levels in 90 min-time point and began to decline. Our experimental setting and that of previous report are not identical, but the expression levels of our studied genes during $60 \mathrm{~min}-$ time period may have shared some important aspects with that study (Kobayashi et al., 2002). Although, our studies were not focused on gene expression that involved in ROS molecules or respiratory burst, one of our studied genes in NADPH oxidase $(C Y B A)$ dictates a partial involvement in phagosome maturation. Our data show that the expression of $C Y B A$ was apparently increased during the first $30 \mathrm{~min}$ of phagocytosis Fig. 4A.

The increasing global concerns over the use of antimicrobial drugs in farmed animals urge us to search for a potential herbal bioactive agent like quercetin, as a feasibility of alternatives to synthesized drugs. From immunological stand point, diets rich in flavonoids, specifically quercetin, have positive health benefits, i.e., reduce infection risk, affect immunity and inflammation. Additionally, solid evidence also suggests that quercetin ultimately benefits in promoting growth and performance and improved feed efficiency in livestock (Gohlke et al., 2013; Maciej et al., 2015; Stoldt et al., 2015).

We speculate that quercetin, in some extents, plays an important role in the regulations at many cellular and molecular levels of bovine neutrophils. Our data herein raises a possibility of the beneficial outcomes of quercetin related to enhancement of bovine neutrophil phagocytosis in combating with pathogenic bacteria. Furthermore, the data in the present study suggest that quercetin further diminishes some proinflammatory cytokines. Our observations are only a starting point for more future research which could result in broadening knowledge of quercetin in bovine neutrophil biology.

\section{Conclusion}

This study provides useful information regarding the use of plant derived flavonoid, quercetin, in two important aspects on bovine neutrophils and could be extended to other innate cell types. Our first finding demonstrates that the use of quercetin during the onset of bacterial infections may enhance the ability of neutrophils to phagocytose invading bacteria. The second, supplementation of quercetin would aid in the anti-inflammation property by attenuating the inflammatory mediators as a consequence of the neutrophil functional response to bacteria. The information in our experiments is worth concluded that quercetin has the ability to boost the expression of genes involved in phagocytosis of bovine neutrophils while suppresses the expression of pro-inflammatory genes.

\section{Acknowledgement}

We would like to thank you the CMU Medical Science Research Equipment Center for flow cytometer. We gratefully acknowledge Christopher C. Salisbury for editing the manuscript.

\section{Funding Information}

This work was financially supported by the FVM, CMU. Grant no. R009071. The authors are also grateful for research funding from the Excellent Center in Veterinary Biosciences (ECVB).

\section{Author's Contributions}

Suphakit Srikok and Sukij Nambut: Execute the study and involve in data acquisition, as well as, analyze and interpret the data. Read and approve the final manuscript. 
Kanruethai Wongsawan: Conceive and design the study, read and approve the final manuscript.

Phongsakorn Chuammitri: Conceive and design the study, execute the study and involve in data acquisition, as well as, analyze and interpret the data. Draft and revise the manuscript. Read and approve the final manuscript.

\section{Disclosure Statement}

We certify that there is no conflict of interest with any financial organization regarding the material discussed in the manuscript.

\section{References}

Amulic, B., C. Cazalet, G.L. Hayes, K.D. Metzler and A. Zychlinsky, 2012. Neutrophil function: From mechanisms to disease. Annu. Rev. Immunol., 30: 459-489.

Bae, H.B., J.W. Zmijewski, J.S. Deshane, J.M. Tadie and D.D. Chaplin et al., 2011. AMP-activated protein kinase enhances the phagocytic ability of macrophages and neutrophils. FASEB J., 25: 4358-4368.

Bao, B.A., C.P. Lai, C.C. Naus and J.R. Morgan, 2012. Pannexin 1 drives multicellular aggregate compaction via a signaling cascade that remodels the actin cytoskeleton. J. Biol. Chem., 287: 8407-8416. DOI: $10.1074 /$ jbc.M111.306522

Berends, E.T., A.R. Horswill, N.M. Haste, M. Monestier and V. Nizet et al., 2010. Nuclease expression by Staphylococcus aureus facilitates escape from neutrophil extracellular traps. J. Innate Immun., 2: 576-586. DOI: 10.1159/000319909

Berger, L., S. Wein, R. Blank, C. Metges and S. Wolffram, 2012. Bioavailability of the flavonol quercetin in cows after intraruminal application of quercetin aglycone and rutin. J. Dairy Sci., 95: 5047-5055. DOI: $10.3168 /$ jds.2012-5439

Bliss, T.W., J.E. Dohms, M.G. Emara and C.L. Keeler, 2005. Gene expression profiling of avian macrophage activation. Vet. Immunol. Immunopathol., 105: 289-299. DOI: 10.1016/j.vetimm.2005.02.013

Boesch-Saadatmandi, C., A. Loboda, A.E. Wagner, A. Stachurska and A. Jozkowicz et al., 2011. Effect of quercetin and its metabolites isorhamnetin and quercetin-3-glucuronide on inflammatory gene expression: Role of miR-155. J. Nutr. Biochem., 22: 293-299. DOI: 10.1016/j.jnutbio.2010.02.008

Boesch-Saadatmandi, C., A.E. Wagner, S. Wolffram and G. Rimbach, 2012. Effect of quercetin on inflammatory gene expression in mice liver in vivo-role of redox factor 1, miRNA-122 and miRNA-125b. Pharmacol. Res., 65: 523-530. DOI: $10.1016 /$ j.phrs.2012.02.007
Boots, A.W., G.R. Haenen and A. Bast, 2008. Health effects of quercetin: From antioxidant to nutraceutical. Eur. J. Pharmacol., 585: 325-337. DOI: 10.1016/j.ejphar.2008.03.008

Brinkmann, V., U. Reichard, C. Goosmann, B. Faulerand Y. Uhlemann et al., 2004. Neutrophil extracellular traps kill bacteria. Science, 303: 1532-1535. DOI: $10.1126 /$ science. 1092385

Cho, S.Y., S.J. Park, M.J. Kwon, T.S. Jeong and S.H. Bok et al., 2003. Quercetin suppresses proinflammatory cytokines production through MAP kinases and NF-kB pathway in lipopolysaccharidestimulated macrophage. Mol. Cell. Biochem., 243: 153-160.

Chuammitri, P., C. Amphaiphan and P. Nojit, 2015. In vitro modulatory effects of quercetin on bovine neutrophil effector functions. Thai J. Vet. Med., 45: 63-72.

Chuammitri, P., S. Srikok, D. Saipinta and S. Boonyayatra, 2017. The effects of quercetin on microRNA and inflammatory gene expression in lipopolysaccharidestimulated bovine neutrophils. Vet. World, 10: 403-410. DOI: 10.14202/vetworld.2017.403-410

De Marchi, U., L. Biasutto, S. Garbisa, A. Toninello and M. Zoratti, 2009. Quercetin can act either as an inhibitor or an inducer of the mitochondrial permeability transition pore: A demonstration of the ambivalent redox character of polyphenols. Biochim. Biophys. Acta Bioenerget., 1787: 1425-1432. DOI: 10.1016/j.bbabio.2009.06.002

Della Libera, A.M.M.P., F.N. De Souza, C.F. Batista, B.P. Santos and L.F.F. De Azevedo et al., 2015. Effects of bovine leukemia virus infection on milk neutrophil function and the milk lymphocyte profile. Vet. Res. DOI: 10.1186/s13567-014-0125-4

Erlund, I., 2004. Review of the flavonoids quercetin, hesperetin and naringenin. Dietary sources, bioactivities, bioavailability and epidemiology. Nutr. Res., 24: 851-874. DOI: 10.1016/j.nutres.2004.07.005

Esmann, L., C. Idel, A. Sarkar, L. Hellberg and M. Behnen et al., 2010. Phagocytosis of apoptotic cells by neutrophil granulocytes: diminished proinflammatory neutrophil functions in the presence of apoptotic cells. J. Immunol., 184: 391-400. DOI: 10.4049/jimmunol.0900564

Formica, J. and W. Regelson, 1995. Review of the biology of quercetin and related bioflavonoids. Food Chem. Toxicol., 33: 1061-1080.

Gohlke, A., C. Ingelmann, G. Nürnberg, A. Starke and S. Wolffram et al., 2013. Bioavailability of quercetin from its aglycone and its glucorhamnosiderutin in lactating dairy cows after intraduodenal administration. J. Dairy Sci., 96: 2303-2313. DOI: $10.3168 /$ jds.2012-6234 
Grinberg, N., S. Elazar, I. Rosenshine and N.Y. Shpigel, 2008. $\beta$-Hydroxybutyrate abrogates formation of bovine neutrophil extracellular traps and bactericidal activity against mammary pathogenic Escherichia coli. Infect. Immun., 76: 2802-2807. DOI: 10.1128/IAI.00051-08

Hoffmann, E., S. Marion, B.B. Mishra, M. John and R. Kratzke et al., 2010. Initial receptor-ligand interactions modulate gene expression and phagosomal properties during both early and late stages of phagocytosis. Eur. J. Cell Biol., 89: 693-704. DOI: 10.1016/j.ejcb.2010.04.006

Jinghui, L., M.A. Marey, R. Kowsar, N. Hambruch and T. Shimizu et al., 2014. An acute-phase protein as a regulator of sperm survival in the bovine oviduct: Alpha 1-acid-glycoprotein impairs neutrophil phagocytosis of sperm in vitro. J. Reprod. Dev., 60: 342-348. DOI: 10.1262/jrd.2014-049

Kennedy, A.D. and F.R. DeLeo, 2009. Neutrophil apoptosis and the resolution of infection. Immunol. Res., 43: 25-61. DOI: 10.1007/s12026-008-8049-6

Keogh, M., T. Spoon, S. Ridgway, E. Jensen and W. Van Bonn et al., 2011. Simultaneous measurement of phagocytosis and respiratory burst of leukocytes in whole blood from bottlenose dolphins (Tursiopstruncatus) utilizing flow cytometry. Vet. Immunol. Immunopathol., 144: 468-475. DOI: $10.1016 /$ j.vetimm.2011.08.017

Khatua, B., K. Bhattacharya and C. Mandal, 2012. Sialoglycoproteins adsorbed by Pseudomonas aeruginosa facilitate their survival by impeding neutrophil extracellular trap through siglec-9. J. Leukoc. Biol., 91: 641-655. DOI: 10.1189/jlb.0511260

Kobayashi, S.D., K.R. Braughton, A.R. Whitney, J.M. Voyich and T.G. Schwan et al., 2003. Bacterial pathogens modulate an apoptosis differentiation program in human neutrophils. Proc. Natl. Acad. Sci. U.S.A., 100: 10948-10953. DOI: $10.1073 /$ pnas. 1833375100

Kobayashi, S.D., J.M. Voyich, C.L. Buhl, R.M. Stahl and F.R. DeLeo, 2002. Global changes in gene expression by human polymorphonuclear leukocytes during receptor-mediated phagocytosis: Cell fate is regulated at the level of gene expression. Proc. Natl. Acad. Sci. U.S.A., 99: 6901-6906.

Kobayashi, S.D., J.M. Voyich, C. Burlak and F.R. DeLeo, 2005. Neutrophils in the innate immune response. Arch. Immunol. Ther. Exp., 53: 505-517.

Liu, J., X. Li, Y. Yue, J. Li and T. He et al., 2005. The inhibitory effect of quercetin on IL-6 production byLPS-stimulated neutrophils. Cell. Mol. Immunol., 2: 455-460.
Livak, K.J. and T.D. Schmittgen, 2001. Analysis of relative gene expression data using real-time quantitative PCR and the $2-{ }^{\Delta \Delta C} \mathrm{~T}$ method. Methods, 25: 402-408. DOI: 10.1006/meth.2001.1262

Maciej, J., C. Schäff, E. Kanitz, A. Tuchscherer and R. Bruckmaier et al., 2015. Bioavailability of the flavonol quercetin in neonatal calves after oral administration of quercetin aglycone or rutin. J. Dairy Sci., 98: 3906-3917. DOI: 10.3168/jds.2015-9361

Martinez, N., L. Sinedino, R. Bisinotto, E. Ribeiro and G. Gomes et al., 2014. Effect of induced subclinical hypocalcemia on physiological responses and neutrophil function in dairy cows. J. Dairy Sci., 97: 874-887. DOI: $10.3168 /$ jds.2013-7408

Nair, M.P., S. Mahajan, J.L. Reynolds, R. Aalinkeel and H. Nair et al., 2006. The flavonoid quercetin inhibits proinflammatory cytokine (tumor necrosis factor alpha) gene expression in normal peripheral blood mononuclear cells via modulation of the NF- $\kappa \mathrm{B}$ system. Clin. Vaccine Immunol., 13: 319-328. DOI: 10.1128/CVI.13.3.319-328.2006

Shirasuna, K., H. Matsumoto, S. Matsuyama, K. Kimura and H. Bollwein et al., 2015. Possible role of interferon tau on the bovine corpus luteum and neutrophils during the early pregnancy. Reproduction, 150: 217-225. DOI: 10.1530/REP-15-0085

Stoldt, A.K., M. Derno, G. Nürnberg, J.M. Weitzel and W. Otten et al., 2015. Effects of a 6-wk intraduodenal supplementation with quercetin on energy metabolism and indicators of liver damage in periparturient dairy cows. J. Dairy Sci., 98: 4509-4520. DOI: $10.3168 /$ jds.2014-9053

Sturn, A., J. Quackenbush and Z. Trajanoski, 2002. Genesis: Cluster analysis of microarray data. Bioinformatics, 18: 207-208.

Valerio, D.A., S.R. Georgetti, D.A. Magro, R. Casagrande and T.M. Cunha et al., 2009. Quercetin reduces inflammatory pain: Inhibition of oxidative stress and cytokine production. J. Nat. Prod., 72: 1975-1979. DOI: $10.1021 / \mathrm{np} 900259 \mathrm{y}$

Yan, J., X. Meng, L.M. Wancket, K. Lintner and L.D. Nelin et al., 2012. Glutathione reductase facilitates host defense by sustaining phagocytic oxidative burst and promoting the development of neutrophil extracellular traps. J. Immunol., 188: 2316-2327. DOI: 10.4049/jimmunol.1102683

Zhang, J., J. Zhu, X. Bu, M. Cushion and T.B. Kinane et al., 2005. Cdc42 and RhoB activation are required for mannose receptormediated phagocytosis by human alveolar macrophages. Mol. Biol. Cell. 16: 824-834. 

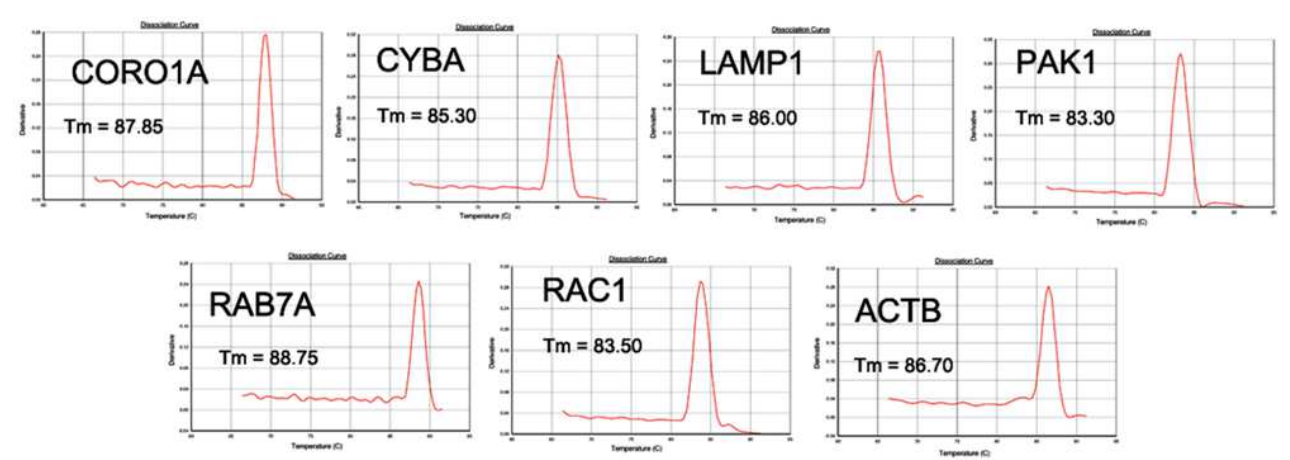

(A)

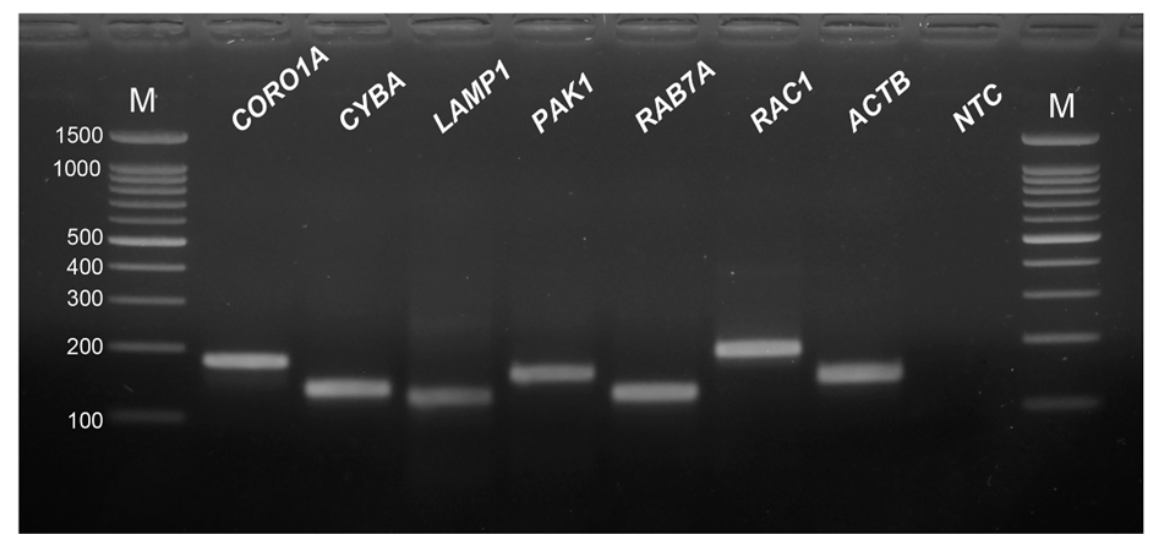

(B)

Suppl. Fig. 1. (A) Representatives of peak in melting profile (dissociation curves) of the real-time PCR reactions. (B) Representative real-time PCR products of expressed genes in bovine neutrophils on agarose gel electrophoresis and EtBr staining. All PCR products had the correct size. $A C T B$ was used as a reference gene. $\mathrm{NTC}=$ no template control, $\mathrm{M}=100 \mathrm{bp}$ DNA marker 